\title{
The Axin/TNKS complex interacts with KIF3A and is required for insulin-stimulated GLUT4 translocation
}

\author{
Hui-Ling Guo ${ }^{1, *}$, Cixiong Zhang ${ }^{1, *}$, Qi Liu ${ }^{1, *}$, Qinxi $\mathrm{Li}^{1}$, Guili Lian ${ }^{1}, \mathrm{Di} \mathrm{Wu}^{1}$, Xuebin $\mathrm{Li}^{1}$, Wei Zhang ${ }^{1}$, \\ Yuemao Shen ${ }^{1,2}$, Zhiyun Ye ${ }^{1}$, Shu-Yong Lin ${ }^{1}$, Sheng-Cai Lin ${ }^{1}$ \\ ${ }^{1}$ State Key Laboratory of Stress Cell Biology, School of Life Sciences, Xiamen University, Xiamen, Fujian 361005, China; ${ }^{2}$ State \\ Key Laboratory of Microbial Technology, School of Life Sciences, Shandong University, Jinan, Shandong 250100, China
}

Insulin-stimulated glucose uptake by the glucose transporter GLUT4 plays a central role in whole-body glucose homeostasis, dysregulation of which leads to type 2 diabetes. However, the molecular components and mechanisms regulating insulin-stimulated glucose uptake remain largely unclear. Here, we demonstrate that Axin interacts with the ADP-ribosylase tankyrase 2 (TNKS2) and the kinesin motor protein KIF3A, forming a ternary complex crucial for GLUT4 translocation in response to insulin. Specific knockdown of the individual components of the complex attenuated insulin-stimulated GLUT4 translocation to the plasma membrane. Importantly, $\mathrm{TNKS}^{-/-}$mice exhibit reduced insulin sensitivity and higher blood glucose levels when re-fed after fasting. Mechanistically, we demonstrate that in the absence of insulin, Axin, TNKS and KIF3A are co-localized with GLUT4 on the trans-Golgi network. Insulin treatment suppresses the ADP-ribosylase activity of TNKS, leading to a reduction in ADP ribosylation and ubiquitination of both Axin and TNKS, and a concurrent stabilization of the complex. Inhibition of Akt, the major effector kinase of insulin signaling, abrogates the insulin-mediated complex stabilization. We have thus elucidated a new protein complex that is directly associated with the motor protein kinesin in insulin-stimulated GLUT4 translocation.

Keywords: Axin; TNKS; KIF3A; GLUT4 translocation

Cell Research (2012) 22:1246-1257. doi:10.1038/cr.2012.52; published online 3 April 2012

\section{Introduction}

Glucose homeostasis is tightly governed by multiple levels of regulation, involving many different molecules, cell types and hormones. GLUT4, one type of glucose transporter, is predominantly expressed in skeletal muscles and adipose tissues, and serves as a major mediator of insulin-stimulated glucose uptake in these tissues [1-4]. In the basal state, GLUT4 is sequestered in intracellular membrane compartments known as GLUT4 storage vesicles (GSVs) formed from either endosomes or the trans-Golgi network (TGN). When blood glucose levels are elevated, insulin secretion is heightened,

*These three authors contributed equally to this work.

Correspondence: Sheng-Cai Lin

Tel: +86-592-2182993; Fax: +86-592-2184687

E-mail: linsc@xmu.edu.cn

Received 5 September 2011; revised 21 December 2011; accepted 22 February 2012; published online 3 April 2012 stimulating glucose uptake into adipocytes and muscle cells by inducing rapid translocation of GLUT4 vesicles to the cell membrane. The main established pathway for insulin-stimulated GLUT4 translocation involves the activation of the insulin receptor and the two downstream kinases PI3K [5, 6] and Akt [7]. Combined depletion of Akt1 and Akt2 markedly attenuates the effect of insulin on GLUT4 movement, in which Akt2 plays a prominent role [8] as mice lacking Akt2 exhibit insulin resistance and a diabetes mellitus-like syndrome [9]. Insulin-mediated GLUT4 translocation is also dependent on the microtubule network $[10,11]$, which motor proteins such as kinesins can use as a track [12] for GSV trafficking. However, the role of microtubule-based cytoskeleton in GLUT4 trafficking remains controversial [13-16]. The kinesin proteins known to participate in anterograde GSV transport include KIF5B [17] and KIF3 [18]. KIF3 consists of KIF3A and KIF3B, forming the kinesin-2 subfamily of the kinesin superfamily, which is linked to cargo vesicles by the kinesin-associated protein [18]. 
The tankyrase (TRF1-interacting ankyrin-related ADP-ribose polymerase, TNKS) protein family consists of two members, tankyrase 1 (TNKS1) and tankyrase 2 (TNKS2), which share $85 \%$ identity in amino-acid sequences [19]. Both proteins contain a poly-ADP-ribosyl polymerase (PARP) domain. TNKS1/2 have been shown to interact with insulin-responsive aminopeptidase (IRAP) [20], which is one of the integral membrane proteins in GLUT4 vesicles [21] and may play important roles in the maintenance and sorting of insulinresponsive GLUT4 vesicles [22, 23]. It was reported that TNKS1 enhanced insulin-stimulated exocytosis of GLUT4 [24]. TNKS1-deficient mice exhibit various metabolic changes including abnormality in glucose metabolism [25], and $\mathrm{TNKS2}^{-/-}$mice show growth retardation [26]. Unfortunately, double-knockout mice are embryonically lethal [19], which has hindered the analysis of the combined roles of both TNKS in glucose homeostasis. Possibility exists that TNKS1 and TNKS2 are functionally redundant to each other, which may explain the uncompromised overall glucose intake in TNKS1deficient mice [25]. Evidence in support of a possible role of TNKS1 in controlling glucose homeostasis also includes that TNKS1 is a candidate gene for diabetes in humans, based on its location in the chromosomal locus of $8 \mathrm{p} 23.1$ that contains susceptibility genes for a subset of monogenic forms of type 2 diabetes [27]. The role of TNKS2 in glucose homeostasis is not clear.

Axin was originally identified as a key inhibiting factor in the Wnt pathway by serving as a central scaffold for the assembly of the degradation complex of $\beta$-catenin [28]. We have identified additional pathways regulated by Axin that are stress-related, including the JNK cascade [29] and DNA damage response [30-32]. Moreover, Axin interacts with DCAP (Drosophila homolog of CAP), a component of the glucose transport regulatory complex, suggesting that Axin may play a role in insulin-mediated glucose transport [33]. Functional linkage between Axin and TNKS came from one study showing that the PARP activity of TNKS mediates PARsylation of Axin and subsequently enhances its ubiquitination and degradation [34]. Down-regulation of Axin by TNKS elevates the level of $\beta$-catenin, leading to the stimulation of Wnt signaling [34].

We have identified, by yeast two-hybrid screens, a series of signaling molecules that interact with Axin, including Daxx, HIPK2 and Pirh2 that are key regulators of the tumor suppressor p53 [30-32]. Among the other clones was the one that encodes tankyrase 2 (TNKS2). To elucidate the biological function of the interaction between Axin and TNKS2, we carried out another round of yeast two-hybrid screen using TNKS2 as the bait. As a result, we identified KIF3A, a kinesin motor protein, as a novel TNKS2-interacting protein. Here we report that Axin, TNKS and KIF3A form a ternary complex that is required for insulin-stimulated GLUT4 redistribution to the cell surface to increase glucose uptake.

\section{Results}

Axin forms a ternary complex with TNKS2 and KIF3A

To search for molecular cues for the physiological function of the Axin/TNKS interaction, a yeast twohybrid screen was carried out using the ankyrin domain (residues 648-937) of TNKS2 as the bait. Interestingly, 19 identified candidate clones encode KIF3A, an essential component of the motor protein kinesin-2. The C-terminal fragment of aa 494-CT is common to all the 19 overlapping KIF3A clones identified. To confirm this interaction, we performed immunoprecipitation (IP) experiments. As shown in Figure 1A, KIF3A was coprecipitated with TNKS by the antibody for endogenous TNKS. We then investigated whether Axin may also bind to KIF3A. Indeed, KIF3A appeared in the immunoprecipitate of Axin, indicating that Axin can also interact with KIF3A (Figure 1B). The TNKS/KIF3A and Axin/ KIF3A interactions were also supported by IP using over-expressed tagged proteins in 293T cells (Supplementary information, Figure S1A). Furthermore, we employed in vitro pull-down assays to examine the direct interaction between TNKS2 and KIF3A, and between Axin and KIF3A. GST-KIF3A could be pulled down by His-tagged TNKS2 (Figure 1C) and by His-tagged Axin (Figure 1D). To address whether Axin, TNKS2 and KIF3A could co-exist in the same complex, we carried out a two-step co-IP, and found that they could form a ternary complex in the cell (Figure 1E). When the three proteins were co-expressed, Axin and KIF3A could be simultaneously detected in the precipitate of FLAGtagged TNKS2 (Supplementary information, Figure S1B). Notably, over-expression of Axin could increase the amount of KIF3 A co-precipitated with TNKS2 (Supplementary information, Figure S1B). This suggests that Axin could promote the association between TNKS2 and KIF3A. We then knocked down Axin by infecting adipocytes with adenovirus-siAxin, and carried out co-IP using the anti-TNKS antibody. As predicted, co-precipitated KIF3A was decreased (Supplementary information, Figure S1C). All these results suggest that, within the ternary complex, each component simultaneously binds to the other two partners, with Axin directly interacting with both TNKS2 and KIF3A, TNKS2 with Axin and KIF3A, and KIF3A with Axin and TNKS2. 


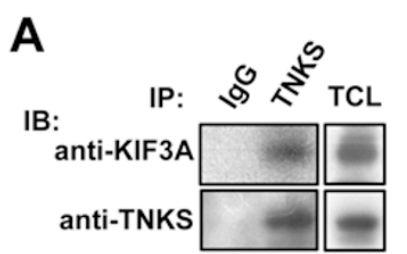

B

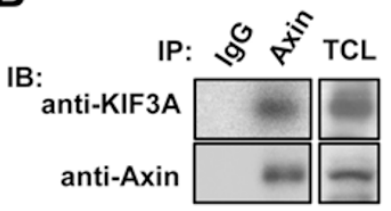

C

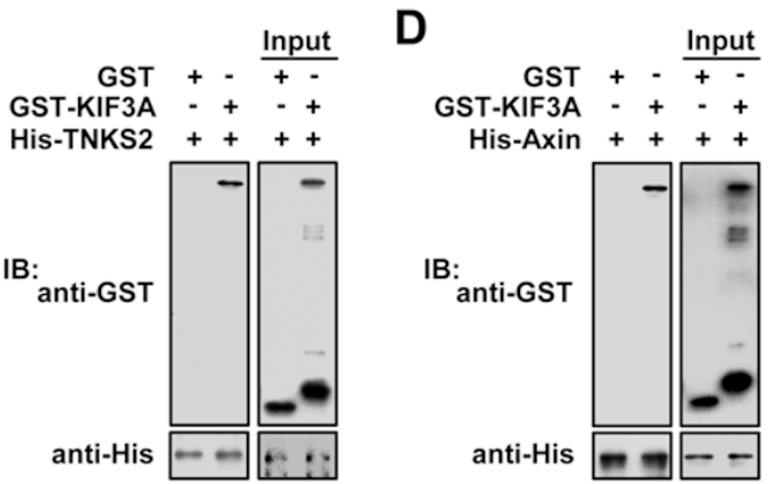

E
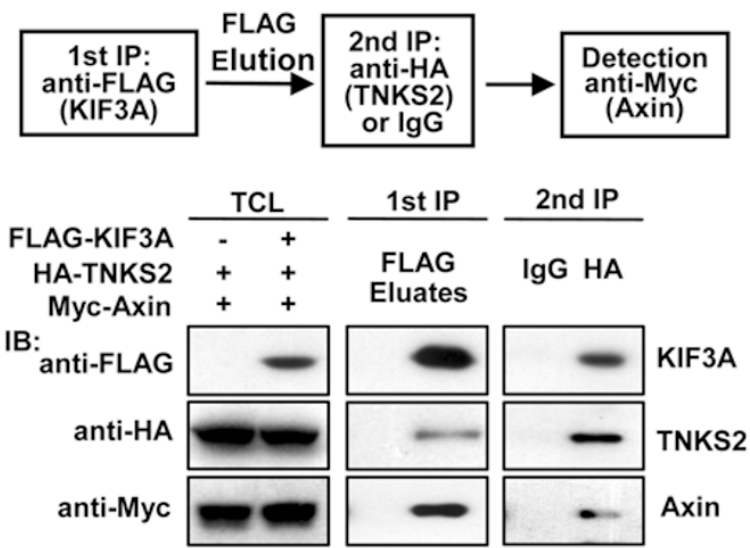

Figure 1 Axin forms a ternary complex with TNKS and KIF3A. (A) TNKS interacts with KIF3A. Lysates of 3T3-L1 adipocytes were subjected to IP with control IgG or rabbit anti-TNKS polyclonal antibody for endogenous TNKS. The immunoprecipitates along with total cell lysates were analyzed by immunoblotting (IB) separately with anti-KIF3A and anti-TNKS antibodies. (B) Axin binds KIF3A. The experiment was performed in the same way as in A, except that anti-Axin antibody was used for IP. (C, D) Interactions between TNKS2 and KIF3A (C) and between Axin and KIF3A (D) were examined by in vitro pull-down assays. Histagged TNKS2 or Axin was incubated with GST or GST-tagged KIF3A for $4 \mathrm{~h}$. The samples were then incubated with Ni-NTA beads to pull down His-tagged TNKS2 or Axin, followed by immunoblotting with anti-His or anti-GST antibody. (E) Axin forms a ternary complex with TNKS2 and KIF3A. HA-TNKS2 and MycAxin were cotransfected with or without FLAG-KIF3A into 293T as indicated beneath TCL. A two-step co-IP was performed as outlined [31]. The FLAG antibody co-precipitated HA-TNKS2 and Myc-Axin only from the cells cotransfected with FLAG-KIF3A.
The Axin/TNKS2/KIF3A complex is critical for GLUT4 translocation in response to insulin

Since KIF3A has been shown to function in microtubule-associated GLUT4 translocation and TNKS1/2 interact with IRAP, an integral protein of GLUT4 vesicles, we set out to determine whether the Axin/TNKS/KIF3A complex participates in insulin-stimulated GLUT4 translocation. First, we carried out immunofluorescent staining in differentiated 3T3-L1 adipocytes as the cells express high levels of GLUT4. We found that Axin, TNKS and KIF3A are co-localized in the perinuclear region of adipocytes (Figure 2A), and that Axin and KIF3A are partially co-localized with the TGN marker TGN38 (Figure 2B). Importantly, TNKS, KIF3A and Axin show co-staining with GLUT4 (Figure 2C). These results indicate that, in the absence of insulin, the Axin/ TNKS/KIF3A complex co-localizes with GLUT4 (Figure $2 \mathrm{C})$. In the presence of insulin, we observed a dramatic redistribution of GLUT4 to the cell surface (Figure 2C). However, insulin treatment did not enrich Axin, TNKS or KIF3A to the cell membrane (Figure 2A-2C, Supplementary information, Figure S2).

We next determined the effects of knockdown of the Axin/TNKS/KIF3A complex on insulin-stimulated translocation of GLUT4 by immunofluorescence microcopy (Figure 3A). The results show that depletion of each component of the Axin/TNKS/KIF3A complex impaired insulin-stimulated GLUT4 translocation, in line with the previous observation that microinjection of anti-KIF3 adaptor subunit (KAP) or anti-KIF3B antibody into adipocytes attenuated GLUT4 translocation [18]. Next, we prepared the plasma membrane fraction of untreated and insulin-stimulated adipocytes that were infected with siRNA-delivering adenoviruses targeting Axin (Figure 3B) or TNKS (Figure 3C) and assessed the relative amounts of the GLUT4 protein in the PM fractions and in the post-PM fractions (supernatants, clear of PM) by immunoblotting. The PM content of GLUT4 after insulin stimulation was drastically decreased in Axinor TNKS-knockdown cells compared to adipocytes expressing control siRNA, whereas the overall expression of GLUT4 was not affected. Insulin receptor $\beta$-subunit (IR- $\beta$ ), which is located on the cell membrane, was detected as a marker for the prepared PM fractions. Double knockdown of TNKS1 and TNKS2 further decreased insulin-stimulated GLUT4 translocation to the PM than single knockdown (Supplementary information, Figure S4). The above data collectively indicate that the complex assembled by Axin, TNKS and KIF3A is important in promoting GLUT4 translocation in response to insulin. 

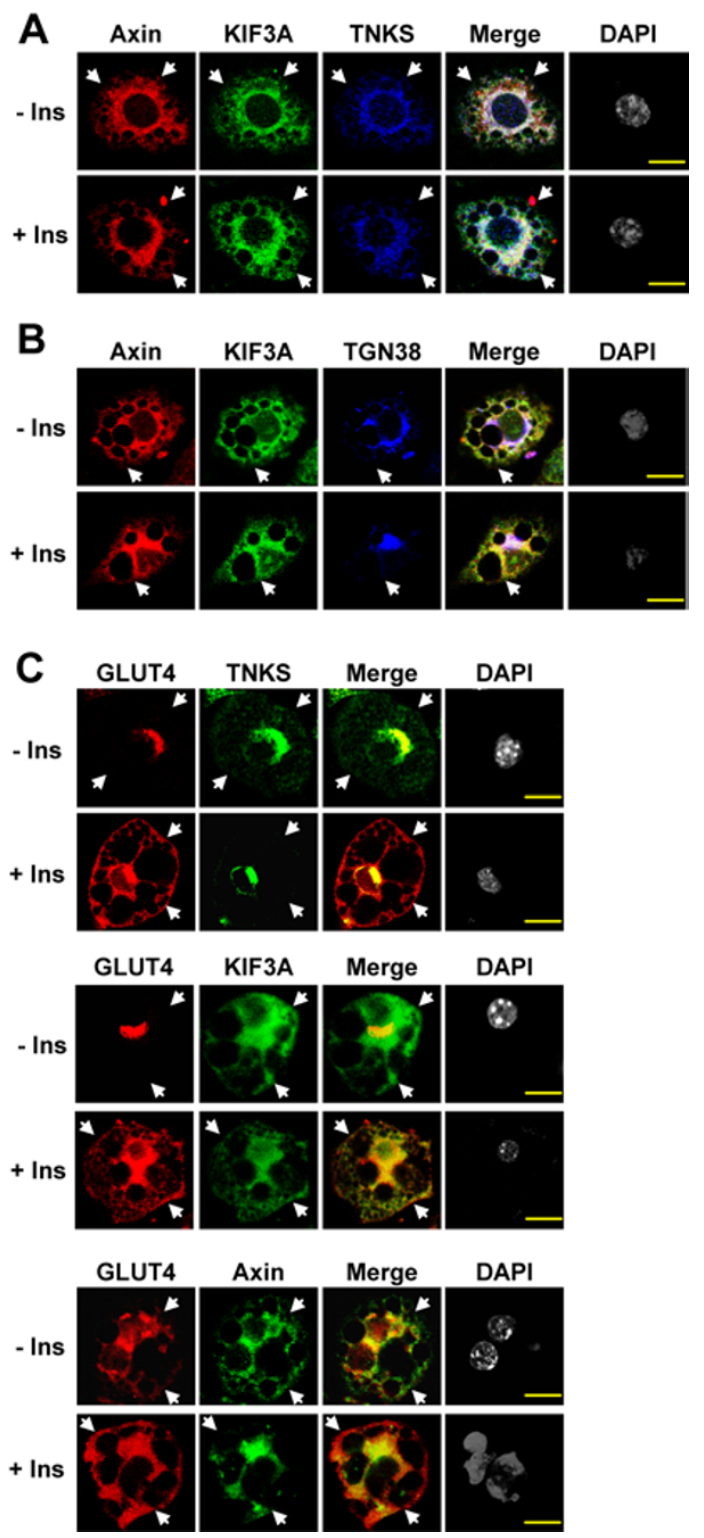

Figure 2 Axin, TNKS and KIF3A are co-localized and highly enriched in the perinuclear region. Differentiated 3T3-L1 adipocytes (d 8) were incubated with or without $200 \mathrm{nM}$ insulin for 30 min after starvation in low-glucose DMEM for $4 \mathrm{~h}$. (A) Cellular Axin, KIF3A and TNKS are co-localized in the perinuclear region. The nuclei were indicated by DAPI staining (gray). Images were acquired with a confocal laser-scanning microscope. After superimposition of images, white color indicates co-localization of Axin (red), KIF3A (green), and TNKS (blue). Arrow heads point to the PM. (B) Axin and KIF3A are partially co-localized with the trans Golgi network marker TGN38. (C) TNKS, KIF3A and Axin have co-localization with GLUT4. Scale bars represent $15 \mu \mathrm{m}$.

Axin/TNKS/KIF3A complex is required for insulin-stimulated glucose uptake

We then assessed whether the Axin/TNKS/KIF3A complex affects glucose uptake by knocking down the expression of Axin, TNKS1/2 or KIF3A individually in 3T3-L1 adipocytes by siRNA-expressing adenoviruses. As can be seen from Figure 4A-4C and Supplementary information, Figure S3, depletion of Axin, KIF3A or TNKS2 attenuated insulin-stimulated 2-deoxyglucose uptake. Re-introduction of each protein by adenoviruses expressing human Axin, TNKS2 and KIF3A restored the insulin-stimulated glucose uptake. Double knockdown of TNKS1 and TNKS2 further decreased insulinstimulated glucose uptake than single knockdown (Figure 4D, Supplementary information, Figure S3C), indicating that TNKS1 and TNKS2 have possibly overlapping functions in the regulation of glucose uptake in adipocytes. Furthermore, we knocked down TNKS2 and KIF3A together and found that the effect on glucose uptake was similar to that of KIF3A single knockdown (Figure 4E), suggesting that TNKS and KIF3A work in conjunction to regulate insulin-stimulated glucose uptake.

We next determined the physiological consequence of TNKS regulation of GLUT4 translocation. We measured the blood glucose levels of adult (12-week-old) wildtype and $T N K S 2^{-/}$mice at normally fed, fasted and re-fed states (Figure 5A), and found that the normally fed and fasted $T N K S 2^{-/}$mice had similar blood glucose levels compared to WT mice. However, the blood glucose levels were significantly higher in the re-fed (fed after fasting) $T N K S 2^{-/-}$mice $(9.06 \pm 0.33 \mathrm{mM})$ compared to wildtype littermates $(7.68 \pm 0.26 \mathrm{mM}, P=0.043)$. The food consumption between wildtype and TNKS2 mice showed no significant difference (Supplementary information, Figure S5). Intriguingly, we observed increased blood glucose levels in normally fed 7-weekold $T N K S 2^{-/-}$mice $(8.33 \pm 0.19 \mathrm{mM})$ compared to WT mice of the same age $(7.2 \pm 0.29 \mathrm{mM}, P=0.012)$ (Figure $5 \mathrm{~B})$. Moreover, glucose tolerance test (GTT) showed that adult $T N K S 2^{-/}$mice had higher levels of blood glucose following administration of an exogenous load of glucose compared with that of WT mice (Figure 5C), suggesting a decreased glucose disposal in the TNKS2-deficient mice. Insulin secretion was similar between the WT and $T N K S 2^{-/-}$mice, at either fasted or fed state (Figure 5D). In fact, in the GTT test, due to elevated blood glucose levels in $T N K S 2^{-/-}$mice, their secreted insulin level was proportionally increased (Supplementary information, Figure S6), indicating that the decreased glucose disposal observed in $\mathrm{TNKS}^{-/-}$mice during the GTT assay is not caused by an abnormal insulin secretion. Following injection of excessive amounts of insulin for the insulin tolerance test (ITT), $T N K S 2^{-/-}$mice showed higher blood glucose levels compared to WT mice (Figure 5E). These results collectively indicate that TNKS2 plays an impor- 
A

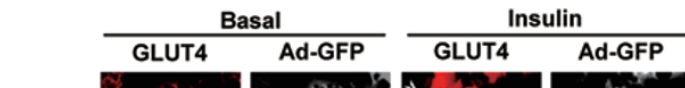

CTL
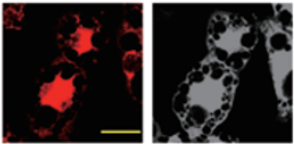

sit1
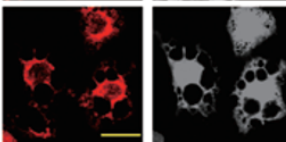

siT2

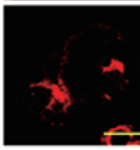

si(T1+T2)

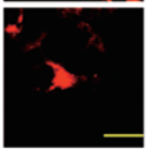

siAxin
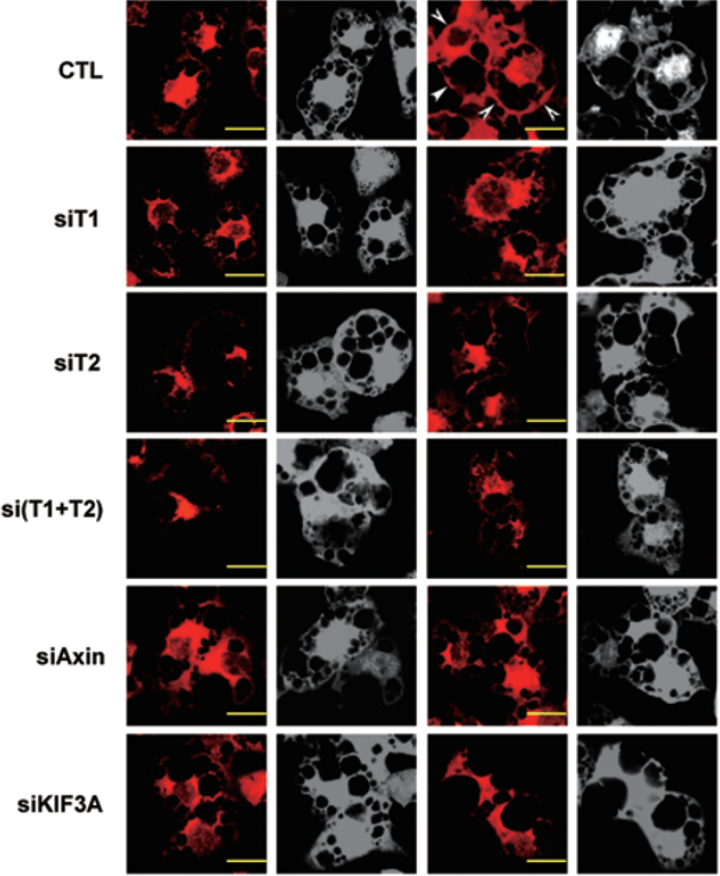

B
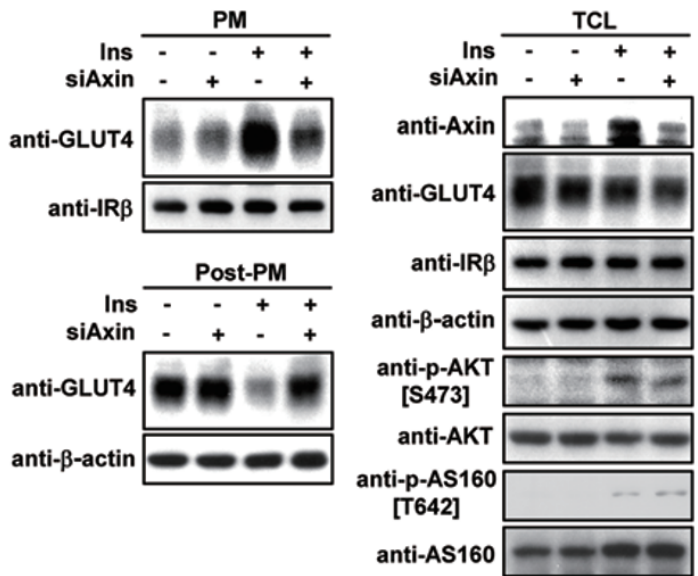

C
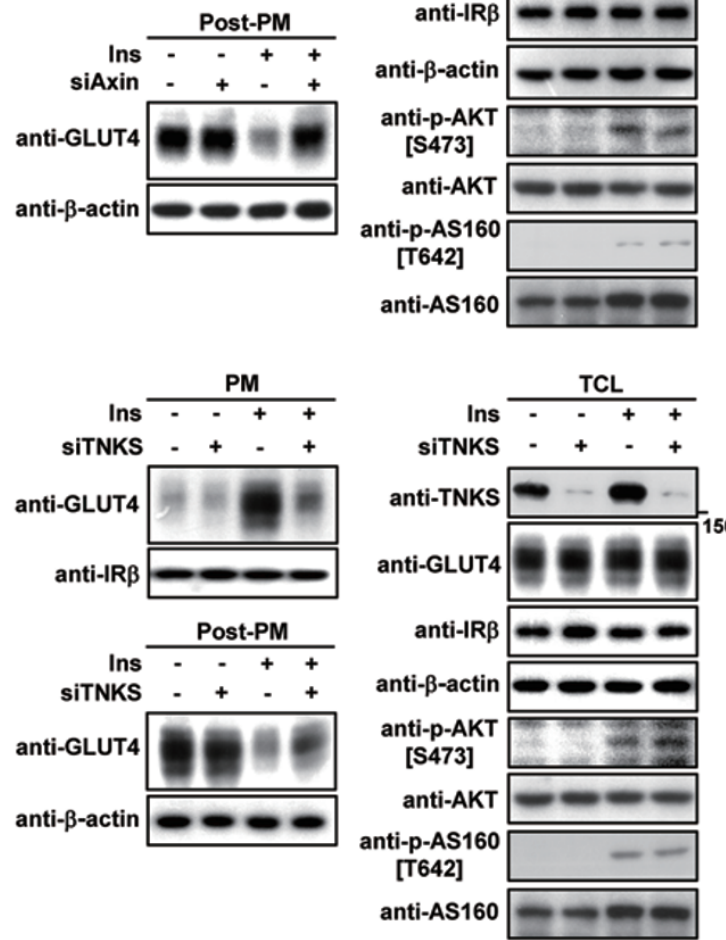
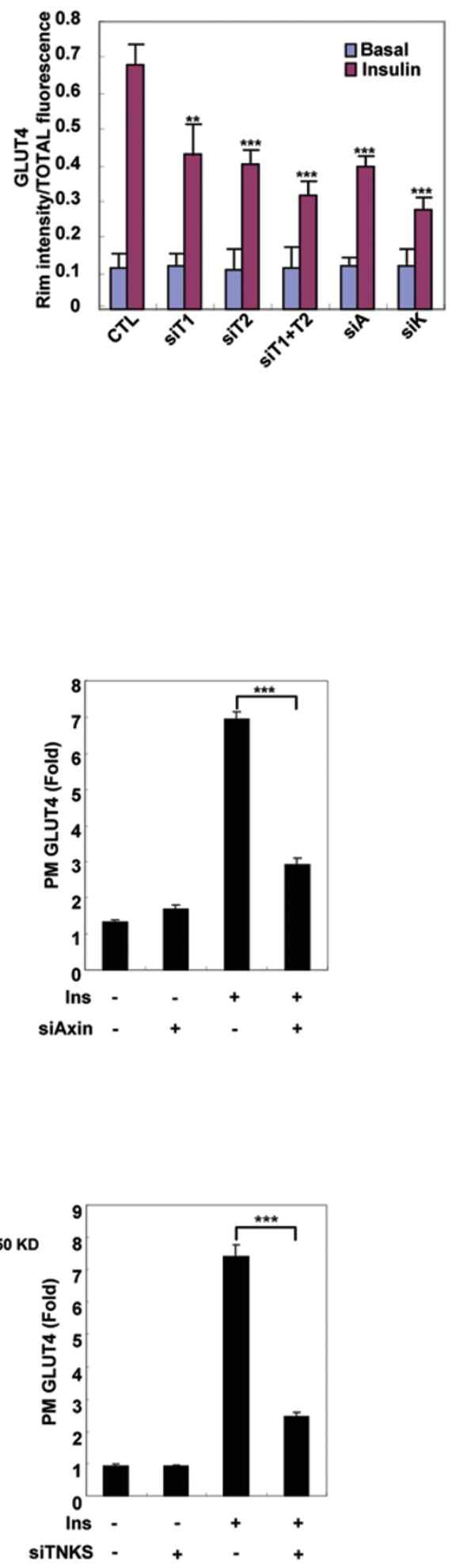
Figure 3 Knockdown of Axin, TNKS or KIF3A impaired GLUT4 translocation. 3T3-L1 cells were induced to differentiate into adipocytes and on day 6 were infected with adenovirus vector (pAdEasy-1) expressing different siRNAs as indicated. (A) Knockdown of Axin, TNKS or KIF3A blocks insulin-dependent GLUT4 translocation. After $48 \mathrm{~h}$ of infection with the indicated siRNA-delivering adenovirus, adipocytes were serum starved for $4 \mathrm{~h}$, followed by incubation with or without $200 \mathrm{nM}$ insulin for $30 \mathrm{~min}$. Cells were then fixed and analyzed by immunofluorescence staining using the GLUT4 antibody and a Texas Redcoupled secondary antibody. Arrow heads point to the PM. The outlines of adipocytes (gray) were displayed by GFP co-expressed from adenovirus. Scale bars represent $20 \mu \mathrm{m}$. The siRNA effects on GLUT4 translocation were re-assessed by Rim intensity/TOTAL fluorescence (Right), which were collected and analyzed using Leica TCS SP2 confocal laser scanning software. The results show that knockdown of each component of the Axin complex attenuates insulin-stimulated GLUT4 translocation to the cell membrane (right, ${ }^{* *} P<0.01$; ${ }^{* * *} P<0.001$ ). (B, C) Knockdown of Axin (B) or TNKS (double knockdown of TNKS1 and TNKS2) (C) attenuates insulin-stimulated GLUT4 translocation to PM. Adipocytes were infected with adenovirus on day 6 and $48 \mathrm{~h}$ later PM fractions were prepared as described in Materials and Methods after insulin (200 nM for 30 min) stimulation. Equal amounts $(15 \mu \mathrm{g})$ of protein of the PM fractions (membrane pellet) and the Post-PM (representing cell supernatant) fractions, along with the total cell lysates (TCL), were subjected to immunoblotting using antibodies indicated. The immunoblots of PM GLUT4 were quantified by densitometry on the right. (Data are mean \pm SEM of three independent experiments. ${ }^{* * *} P<0.001$.)

tant role in mediating insulin-stimulated glucose uptake in vivo.

Insulin stabilizes Axin/TNKS/KIF3A complex by suppressing TNKS PARsylase activity

We next assessed whether the Axin and TNKS pro- teins are dynamically modulated before and after insulin treatment. As shown in Figure 6A, insulin treatment increased cellular levels of Axin and TNKS, which is not due to altered mRNA abundance as indicated by quantitative RT-PCR analysis (Supplementary information, Figure S7A and S7B). Since TNKS1/2 PARsylate them-
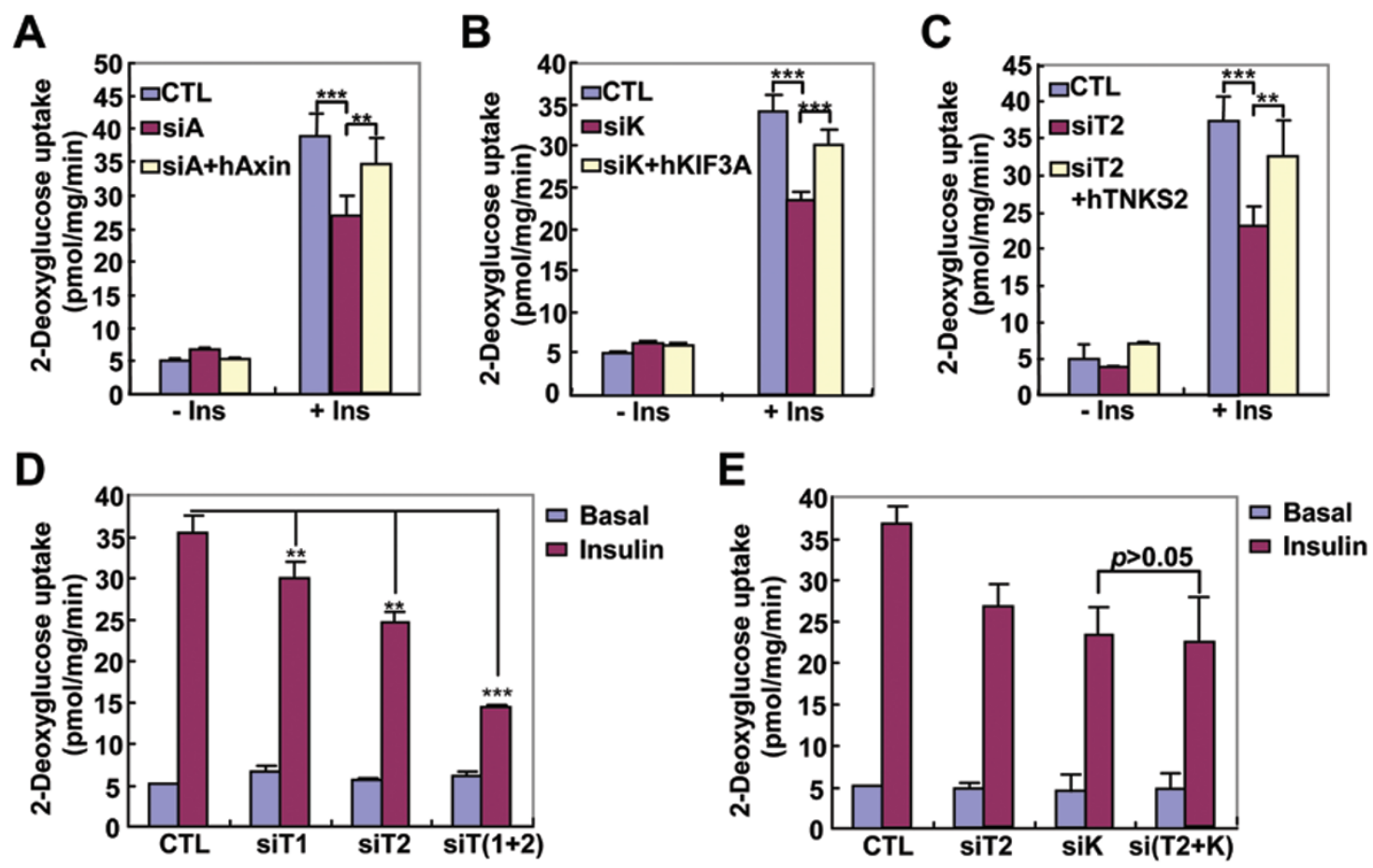

Figure 4 Knockdown of components of the Axin complex attenuated insulin-stimulated glucose uptake. Single knockdown of Axin (siA) (A), KIF3A (siK) (B) or TNKS2 (siT2, ${ }^{* *} P<0.01$; $\left.{ }^{* *} P<0.001\right)(\mathrm{C})$, and double knockdown of TNKS1 and TNKS2 (D) or KIF3A and TNKS2 (E) were carried out, respectively. 3T3-L1 cells were induced to differentiate into adipocytes and on day 6 were infected with adenovirus vector (pAdEasy-1) expressing different siRNAs as indicated. Experiments were performed $48 \mathrm{~h}$ after infection according to the protocol described in Materials and Methods. 
A
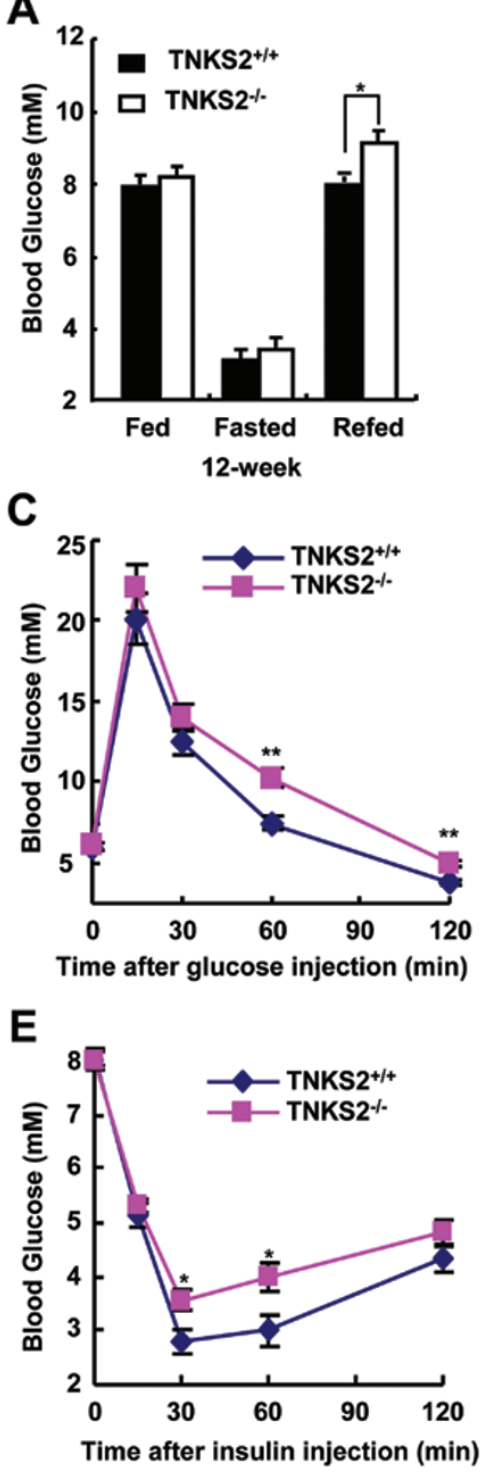

Figure 5 TNKS2 is required in insulin-stimulated glucose disposal. (A) Analysis of blood glucose concentrations in normal and mutant adult mice (12-week males). Mice $\left(T N K S 2^{+/+}, n=6\right.$; $T N K S 2^{-1-}, n=5$ ) were fasted for $16 \mathrm{~h}$, and re-fed for $1 \mathrm{~h}$. Blood glucose levels in the fasted mice and re-fed mice were separately determined. (B) Blood glucose levels of young (7-week) males $\left(T N K S 2^{+/+}, n=7 ; T N K S 2^{-/-}, n=8\right)$ were determined, showing a significant difference between the WT and mutant mice ( $\left.{ }^{*} P<0.05\right)$. (C) Glucose tolerance test. Animals were fasted for $16 \mathrm{~h}$. D-Glucose $(2 \mathrm{~g} / \mathrm{kg})$ was administrated into mice by intraperitoneal injection, and blood glucose concentrations were determined at the indicated times (12-week male mice; $T_{N K S 2}^{+/+}, n=6,24-27 \mathrm{~g} ; \mathrm{TNKS2}^{-/-}, n=5,20-24 \mathrm{~g} ;{ }^{*} P<0.05$, $\left.{ }^{* *} P<0.01\right)$. (D) WT $(n=13)$ and mutant mice $(n=12)$ were fed or fasted overnight, and their insulin levels were determined. (E) Insulin tolerance test. $1 \mathrm{U} / \mathrm{kg}$ human insulin was administered into 12-week mice $\left(\right.$ TNKS2 $^{+/+}, n=6,24-27 \mathrm{~g}$; $\mathrm{TNKS2}^{-/-}, n=5$, 20-24 g) by intraperitoneal injection and blood glucose levels were determined at the indicated times. selves and Axin, increasing their ubiquitination and subsequent degradation [34], we next determined the extents of PARsylation and ubiquitination of TNKS and Axin in the presence or absence of insulin. 3T3-L1 adipocytes were serum-starved for $4 \mathrm{~h}$, followed by insulin treatment in the presence of MG132 (Figure 6B). Axin and TNKS proteins were then immunoprecipitated and the effects of insulin on their PARsylation and ubiquitination were separately monitored by immunoblotting using anti-PAR and anti-ubiquitin (Ub) antibodies. The results showed that insulin treatment obviously reduced both PARsylation and ubiquitination of TNKS and Axin, indicating that the TNKS PARsylase activity was suppressed upon insulin stimulation. We also checked whether GLUT4 is PARsylated or ubiquitinated with or without the treatment of insulin. The results show that GLUT4 is indeed PARsylated and ubiquitinated. However, the PARsylation and ubiquitination as well as the total GLUT4 protein levels are not affected by insulin treatment (Supplementary information, Figure S8).

We then tested whether levels of the Axin/TNKS/KIF3A complex formation were changed with addition of insulin by carrying out co-IP of the endogenous complex proteins from 3T3-L1 adipocytes treated with or without insulin with the antibody against TNKS. Increased amounts of KIF3A and Axin were co-precipitated with TNKS in cells treated with insulin (Figure $6 \mathrm{C}$ ). We also investigated Axin/TNKS/KIF3A complex formation in C2C12 cells through co-IP (Supplementary information, Figure S9), which showed that the complex formation is conserved between adipocytes and muscle cells.

To support the notion that the Axin/TNKS/KIF3A complex is stabilized by insulin through inhibition of TNKS PARsylase activity, we synthesized the TNKS PARsylase inhibitor XAV939. As shown in Supplementary information Figure S10A, XAV939 could promote the tripartite complex formation. Furthermore, XAV939 could increase insulin-stimulated glucose uptake by about 30\% (Supplementary information, Figure S10B) and enhance insulin-simulated GLUT4 translocation to the PM (Supplementary information, Figure S10C). Together, these findings suggest that insulin stimulation can enhance the Axin/TNKS/KIF3A complex formation by suppressing TNKS-mediated PARsylation and subsequent ubiquitination/degradation of Axin and TNKS itself.

Akt mediates insulin-stimulated Axin/TNKS/KIF3A complex stabilization

To further analyze the direct involvement of insulin signaling in modulating the complex of Axin/TNKS/ KIF3A, we determined the effects of Akt inhibition on 
A

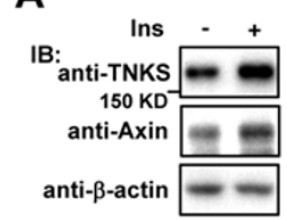

B
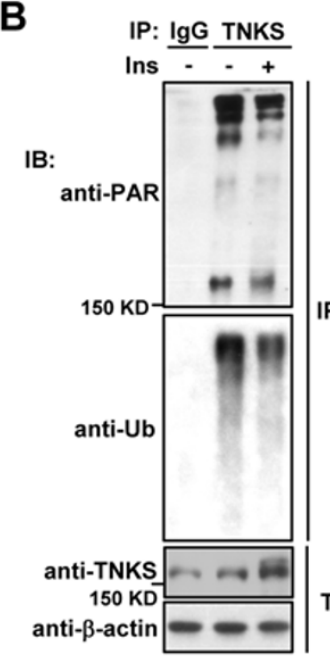

C
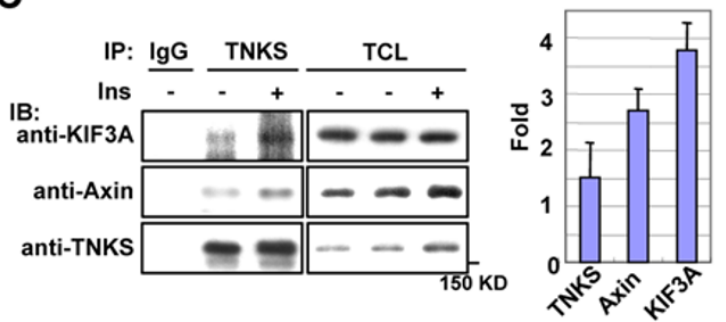

Figure 6 Insulin stabilizes the Axin/TNKS/KIF3A complex by suppressing TNKS PARsylation activity. (A) Insulin treatment increases protein levels of TNKS and Axin. Differentiated 3T3L1 adipocytes were treated with or without $200 \mathrm{nM}$ insulin for $30 \mathrm{~min}$ after $4 \mathrm{~h}$ serum deprivation, and were harvested with SDS sample buffer for western blotting with antibodies for endogenous TNKS and Axin. The bar graphs show basal (lane 1) and insulin-stimulated (lane 2) TNKS and Axin protein levels (normalized to lane 1) determined from immunoblotting. (B) PARsylation and ubiquitination of TNKS (left panel) and Axin (right panel) were decreased after insulin treatment. Adipocytes were serum starved for $4 \mathrm{~h}$, and then incubated with DMEM supplemented with or without $200 \mathrm{nM}$ insulin for $30 \mathrm{~min}$ in the presence of $20 \mu \mathrm{M}$ MG132. The endogenous TNKS proteins were immunoprecipitated with anti-TNKS, followed by analysis of their levels of ADP-ribosylation and ubiquitination using antipoly-ADP-ribose (PAR) and anti-ubiquitin antibodies, respectively. (C) Insulin treatment elevates the abundance of the Axin/TNKS/ KIF3A complex. Adipocytes were serum-depleted for $4 \mathrm{~h}$, then added with or without insulin to a final concentration of $200 \mathrm{nM}$, and incubated for $30 \mathrm{~min}$ before lysis. The endogenous proteins were immunoprecipitated with anti-TNKS antibody, followed by immunoblotting with individual antibodies to determine levels of each protein in the complex, quantified by densitometry after normalizing to their respective levels from the unstimulated cells. the abundance of these proteins and on their interaction in cells treated with or without insulin by IP and western blotting analysis. As can be seen from Figure 7A, while insulin treatment increased the protein levels of Axin and TNKS, and the co-IP of KIF3A with TNKS, Akt inhibitor VIII (for both Akt1 and Akt2) abrogated such increases. Mechanistically, Akt acts to suppress PARsylase activity of TNKS, because treatment with the Akt inhibitor reversed the effect of insulin on PARsylation and subsequent ubiquitination of TNKS (Figure 7B). Moreover, we carried out co-IP experiments to check if insulin treatment could increase the association of the complex with microtubules and GLUT4 vesicles. Increased levels of TNKS were detected in the GLUT4 immunoprecipitate (Supplementary information, Figure S11), and tubulin and KIF3A were more abundantly co-present in the TNKS immunoprecipitate (Supplementary information, Figure S12), from insulin-treated cells.

\section{Discussion}

In the present study, we have demonstrated that Axin, TNKS and KIF3A form a multimeric complex that mediates insulin-stimulated GLUT4 translocation and glucose uptake. Remarkably, both Axin and TNKS are directly linked to KIF3A, a critical component of KIF3 known to be necessary for GLUT4 translocation. We have also provided multiple lines of evidence to demonstrate that this complex is directly involved in glucose transport by promoting GLUT4 translocation, at least in part from the Golgi origin, to the surface membrane in response to insulin. First of all, although normally fed adult $\mathrm{TNKS}^{-/-}$mice show little change in blood glucose levels, the normally fed young $T N K S 2^{-/-}$mice have significantly higher blood glucose levels compared to the wildtype littermates. Second, adult re-fed TNKS2 $2^{-1-}$ mice display a significant increase in blood glucose levels. GTT and ITT assays also show that the decline of blood glucose in $\mathrm{TNKS2}^{-/-}$mice was slower following injection of excessive glucose or insulin. When Axin, TNKS and KIF3A were knocked down individually by siRNA in 3T3-L1 adipocytes, insulin-induced GLUT4 translocation and glucose uptake were obviously impaired. We knocked down TNKS and KIF3 together and found that the effect on glucose uptake was similar to that of KIF3A single knockdown, suggesting that TNKS and KIF3A work in conjunction to regulate insulinstimulated glucose uptake. Third, immunofluorescence staining experiments revealed co-localization of the complex components with GLUT4 as well as with the Golgi apparatus in the perinuclear region. TNKS has been shown to interact with IRAP [20], further suggest- 

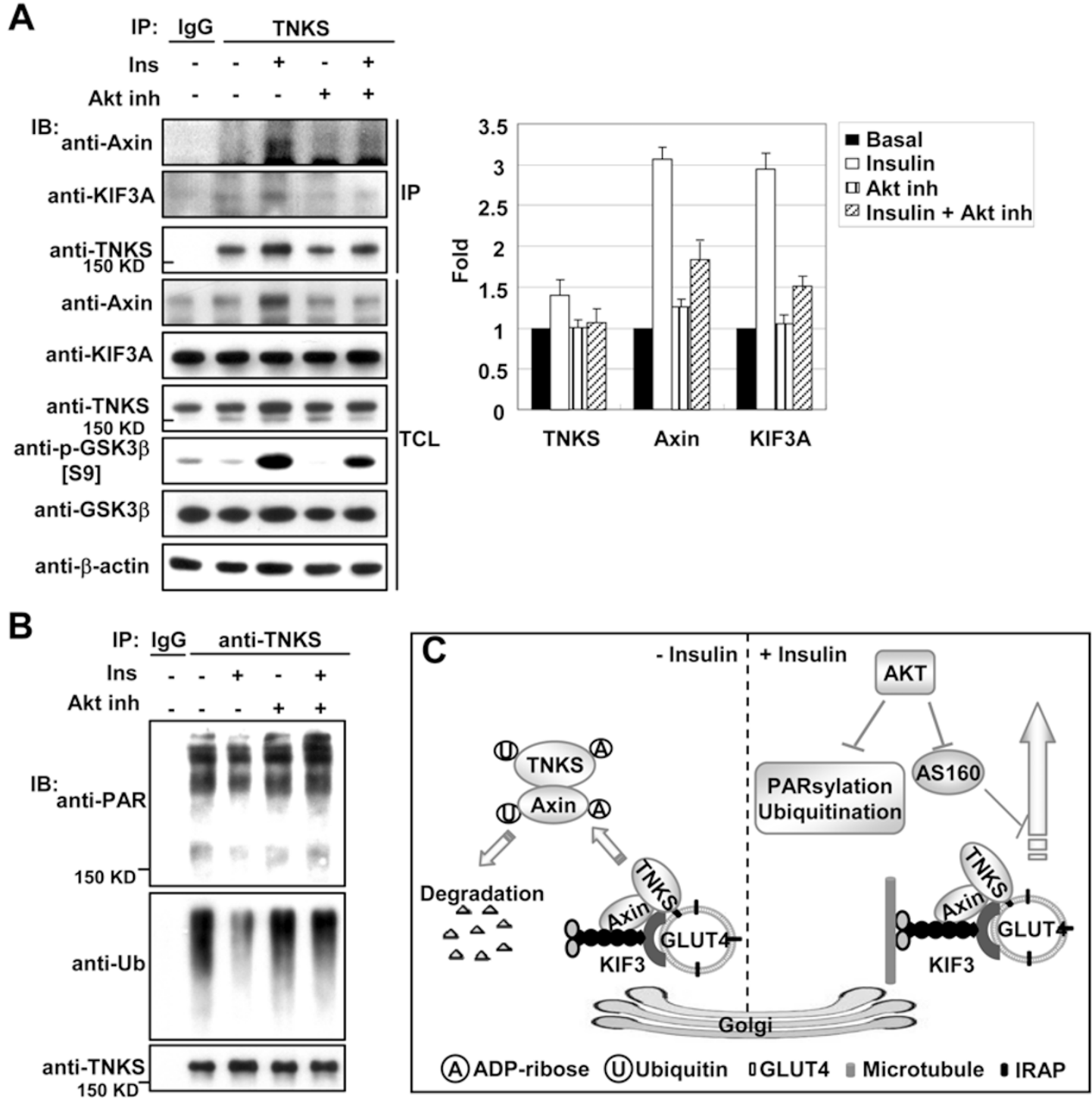

Figure 7 Akt dependence of insulin-induced accumulation of the Axin/TNKS/KIF3A complex. (A) Inhibition of Akt attenuates insulin-induced complex formation. 3T3-L1 cells were serum starved for $4 \mathrm{~h}$ and then incubated with $1.8 \mu \mathrm{M}$ Akt inhibitor VIII (inh) and $200 \mathrm{nM}$ insulin (ins) for $30 \mathrm{~min}$, followed by IP with anti-TNKS antibody. The bar graphs (on the right) show fold increases of indicated protein on lanes 2-5 (normalized to lane 2). The precipitated TNKS and the co-precipitated proteins were analyzed by immunoblotting using specific antibodies as indicated. (B) Inhibition of Akt antagonizes the effect of insulin on TNKS PARsylation and ubiquitination. Insulin treatment decreases PARsylation and ubiquitination of TNKS (lane 3 compared to lane 2), which was abrogated by the Akt inhibitor. (C) A simplified model showing that the Axin/TNKS complex is connected to GLUT4 vesicles trafficking by KIF3A. Axin, TNKS and KIF3A form a ternary complex that is associated with the TGN. In the absence of insulin, TNKS and Axin are degraded after poly-ADP-ribosylation and ubiquitination. Insulin treatment prevents the PARsylation of TNKS and Axin in an Akt-dependent manner to increase the abundance of the Axin/TNKS/KIF3A complex, in that Axin acts to enhance the complex. It has been shown that insulin-activated Akt phosphorylates AS160 that is a GTPase-activating protein for Rabs; upon phosphorylation, AS160 can no longer exert its inhibitory effect on Rabs, ultimately allowing GSV to translocate to the cell surface [37-42].

ing a functional linkage of the Axin/TNKS complex to glucose transport.

It is worthy to note that insulin signaling diverges to intersect with GLUT4 trafficking via multiple routes.
Both PI3K-dependent and PI3K-independent pathways operate in the insulin-induced GLUT4 translocation [1, 4]. Genetic or pharmacologic disruption of Akt inhibits only about two-thirds of the signaling to GLUT4 $[8,35$, 
36]. Since the Axin/TNKS/KIF3A complex is regulated in an AKT-dependent manner, the partial effect of the TNKS2 knockout on glucose disposal is therefore not unexpected. In particular, we have provided evidence supporting a functional overlap between TNKS1 and TNKS2, as shown in glucose uptake assays.

Based on the data from our current study and from others, it is clear that in the absence of insulin, TNKS proteins poly-ADP-ribosylate themselves and Axin, leading to their degradation. Insulin signaling suppresses the PARsylase activity of TNKS in an Aktdependent manner to stabilize the Axin/TNKS/KIF3A complex. By virtue of its simultaneous interaction with both TNKS and KIF3A, Axin serves as a scaffold to facilitate the formation of the ternary complex in the presence of insulin, which may promote the complex to associate with GLUT4 vesicles to enable their ultimate translocation to the PM (Figure 7C). The observation that treatment with the TNKS PARsylase inhibitor XAV939 could increase the complex formation, GLUT4 translocation, and insulin-stimulated glucose uptake further underscores the importance of PARsylase activity in the regulation of glucose uptake. Together, we have uncovered a functional linkage between the Axin/TNKS complex and the kinesin motor for GLUT4 translocation in response to insulin, in which the PARsylase activity plays a negative role in controlling the stability of the translocation complex. However, it remains unclear how insulin-activated Akt signals to the Axin complex to trigger GLUT4 translocation to the cell surface. It has been shown that Akt can phosphorylate AS160, leading to derepression of the small G protein Rabs for GLUT4 trafficking [37-42]. The functional interrelationship between the Axin/TNKS/KIF3A complex and Akt-mediated regulation of AS160 awaits further investigation.

\section{Materials and Methods}

\section{Plasmid constructions}

CMV2-FLAG-TNKS2 (human) was kindly provided by Dr Chi NW. It was tagged with three tandem HA epitopes fused to the N-terminus and cloned to pCMV5 vector. Full-length cDNA encoding mouse KIF3A was obtained from an EST clone (IMAGE No. 601205534, Open Biosystem).

\section{RNA interference and adenovirus infection}

Adenovirus vector pAdEasy (GFP) was used for expression of siRNA. mTNKS1: 5'-GAGATGCAGAGCACTATTC-3'; mTNKS2: 5'-GGAAGGATGTAGTTGAAT A-3'; mAxin1: 5'-GGAGAGAAGGAGATCAGTA-3'; mKIF3A: 5'-AGCTAAAATGTGTGG CCTA-3'. 3T3-L1 cells were induced to differentiate into adipocytes and on day 6 were infected with individual adenovirus. After $48 \mathrm{~h}$ of infection, adipocytes were subjected into experiments.

\section{Cell culture}

293 T and 3T3-L1 cells were maintained in Dulbecco's modified Eagle's medium (DMEM) supplemented with 10\% fetal bovine serum, $2 \mathrm{mM}$ L-glutamine, $100 \mathrm{IU}$ penicillin and $100 \mathrm{mg} /$ $\mathrm{ml}$ streptomycin at $37^{\circ} \mathrm{C}$ in a humidified incubator containing $5 \%$ $\mathrm{CO}_{2}$. Differentiation of 3T3-L1 cells was initiated at day 2 postconfluence by addition of $5 \mu \mathrm{g} / \mathrm{ml}$ of insulin, $1 \mu \mathrm{M}$ dexamethasone (DEX), and 0.5 mM 3-isobutyl-1-methylxanthine (IBMX) for $2 \mathrm{~d}$, followed by culturing in DMEM supplemented with 10\% FBS and $5 \mu \mathrm{g} / \mathrm{ml}$ insulin for additional $2 \mathrm{~d}$. The differentiated cells were then maintained in normal culture medium.

\section{In vivo PARsylation}

cells were serum starved in low glucose DMEM ( $1 \mathrm{~g} / 1$ glucose, Hyclone) for $4 \mathrm{~h}$, and then treated with $20 \mu \mathrm{M}$ MG132 accompanied with or without $200 \mathrm{nM}$ insulin for $30 \mathrm{~min}$. Cells were harvested with RIPA buffer $(50 \mathrm{mM}$ Tris- $\mathrm{HCl}, \mathrm{pH} 7.4,150 \mathrm{mM}$ $\mathrm{NaCl}, 1 \% \mathrm{NP}-40,0.5 \%$ sodium deoxycholate, 1 mM EDTA, $1 \mu \mathrm{M}$ leupeptin, $1 \mu \mathrm{M}$ PMSF) supplemented with $1 \%$ SDS. Cleared cell lysates were diluted with RIPA buffer to reduce the SDS concentration to $0.2 \%$ and subjected to IP using the antibodies indicated and protein $\mathrm{A} / \mathrm{G}$ beads overnight, followed by western blotting.

\section{Yeast two-hybrid screen}

Yeast two-hybrid screen of a mouse brain cDNA library was carried out using MATCHMAKER GAL4 Two-Hybrid System 3 (Clontech) according to the manufacturer's instructions as described previously [31]. The sequence of aa 648-937 encompassing the ankyrin-repeat and SAM domain of human tankyrase 2 (TNKS2) was used as the bait.

\section{Antibodies and drugs}

Rabbit polyclonal anti-Axin antibodies $\mathrm{C} 2 \mathrm{~b}$ and $\mathrm{C} 8 \mathrm{~b}$ were raised as described previously [30]. Commercial antibodies used in this study include mouse anti-HA (F-7), anti-Myc (9E10), goat anti-Axin (S-20), rabbit anti-tankyrase1/2 (H350), goat antiGLUT4 (C20), rabbit anti-TGN38 (M-290) and mouse anti-ubiquitin (P4D1) (Santa Cruz Biotechnology), rabbit anti-GLUT4 antibody (ab654, Abcam), mouse anti-FLAG antibody (M2 antibody, Sigma), mouse anti-KIF3A (BD Transduction Laboratories), and rabbit anti-KIF3A antibody (Abcam). Bovine insulin,IBMX, and DEX were purchased from Sigma, and Akt inhibitors VIII from Calbiochem.

\section{Immunofluorescence staining}

3T3-L1 cells were differentiated to adipocytes and plated on 22- $\mathrm{mm}^{2}$ coverslips in six-well plates. After serum starvation for 4 $\mathrm{h}$, adipocytes were treated with or without $200 \mathrm{nM}$ insulin for 30 min, followed by fixation as previously described [32]. Cells were then incubated with indicated primary antibodies in blocking solution overnight at $4{ }^{\circ} \mathrm{C}$. Next, cells were washed four times with PBS containing $0.02 \%$ Triton X-100, 1.5\% BSA and $1 \mathrm{mM} \mathrm{NaN}_{3}$, incubated with $4 \mu \mathrm{g} / \mathrm{ml}$ of bovine anti-goat Texas red, donkey anti-mouse Alexa Fluor 488, goat anti-rabbit Alexa Fluor 647 in blocking solution for $2 \mathrm{~h}$. Cells were then washed and stained with DAPI $(1 \mu \mathrm{g} / \mathrm{ml})$ in PBS for $5 \mathrm{~min}$.

\section{Glucose uptake assay}

3T3-L1 cells grown in 12-well plates and were induced to 
differentiate to adipocytes. Mature adipocytes ( $8 \mathrm{~d}$ ) were serum starved for $4 \mathrm{~h}$, incubated with or without $200 \mathrm{nM}$ insulin for 20 min and then subjected to a 2-deoxy-D-glucose (2-DG) $(0.25 \mu \mathrm{Ci}$ ${ }^{3} \mathrm{H}-2 \mathrm{DG}$ per well) (Perkin Elmer). 10 min later, after three times washes, cells were lysed and intracellular 2-DG, which cannot be metabolized, was measured by liquid scintillation counting.

\section{Plasma membrane isolation}

According to the protocol described in [43], 3T3-L1 adipocytes were collected and broken by passing through a 25 -gauge needle in a hypotonic buffer (50 mM Tris-HCl, $\mathrm{pH} 8.0,0.1 \%$ NP-40, 0.5 $\mathrm{mM}$ DTT, protease inhibitors and phosphatase inhibitors). After centrifugation at $1000 \times \mathrm{g}$ for $10 \mathrm{~min}$, the supernatant was collected. The pellet was washed two times with hypotonic buffer without NP-40 and lysed in a buffer (50 mM Tris-HCl, $\mathrm{pH} 8.0,1 \%$ NP-40, $0.5 \mathrm{mM}$ DTT, protease inhibitors and phosphatase inhibitors) for $1 \mathrm{~h}$ at $4{ }^{\circ} \mathrm{C}$. The re-suspended pellet was then centrifuged at $16000 \times \mathrm{g}$ for $20 \mathrm{~min}$. The supernatant was collected as the PM fraction. The supernatant collected from the first centrifugation was cleared by centrifugation at $16000 \times g$ for $20 \mathrm{~min}$, and the resulting supernatant was collected as Post-PM fraction.

\section{GTT and ITT}

For GTT, mice after overnight fast $(16 \mathrm{~h})$ were intraperitoneally injected with D-Glucose $(2 \mathrm{~g} / \mathrm{kg})$, and blood glucose concentrations were determined at $0,15,30,60,120$ min after injection. In ITT, animals that had been fasted for $4 \mathrm{~h}$ were administrated by intraperitoneal injection of human insulin $(1 \mathrm{U} / \mathrm{kg})$ and blood glucose levels were determined at the indicated times with Accucheck (Roche).

\section{Acknowledgments}

We thank Dr Chi NW for providing the CMV2-FLAG-TNKS2 (human) construct. This work was supported by grants from the National Basic Research Program of China (2011CB910800 to SCL), the National Natural Science Foundation of China (31130016 to SCL, 30921005 to SCL, and 30970613 to QL), NCET-09-0675 (to QL), and the Science Planning Program of Fujian Province (2009J06021 to QL).

\section{References}

1 Bryant NJ, Govers R, James DE. Regulated transport of the glucose transporter GLUT4. Nat Rev Mol Cell Biol 2002; 3:267-277.

2 Hou JC, Pessin JE. Ins (endocytosis) and outs (exocytosis) of GLUT4 trafficking. Curr Opin Cell Biol 2007; 19:466-473.

3 Klip A. The many ways to regulate glucose transporter 4. Appl Physiol Nutr Metab 2009; 34:481-487.

4 Leney SE, Tavare JM. The molecular basis of insulinstimulated glucose uptake: signalling, trafficking and potential drug targets. J Endocrinol 2009; 203:1-18.

5 Martin S, Haruta T, Morris A, Klippel A, Williams L, Olefsky J. Activated phosphatidylinositol 3-kinase is sufficient to mediate actin rearrangement and GLUT4 translocation in 3T3-L1 adipocytes. J Biol Chem 1996; 271:17605-17608.

6 Clarke JF, Young PW, Yonezawa K, Kasuga M, Holman JD.
Inhibition of the translocation of GLUT1 and GLUT4 in 3T3L1 cells by the phosphatidylinositol 3-kinase inhibitor, wortmannin. Biochem J 1994; 300:631-635.

$7 \mathrm{Ng}$ Y, Ramm G, Lopez, JA, James DE. Rapid activation of Akt2 is sufficient to stimulate GLUT4 translocation in 3T3-L1 adipocytes. Cell Metab 2008; 7:348-356

8 Jiang ZY, Zhou QL, Coleman KA, Chouinard M, Boese Q, Czech MP. Insulin signaling through Akt/protein kinase B analyzed by small interfering RNA-mediated gene silencing. Proc Natl Acad Sci USA 2003; 100:7569-7574.

9 Cho H, Mu J, Kim JK, Thorvaldsen JL, et al. Insulin resistance and a diabetes mellitus-like syndrome in mice lacking the protein kinase Akt2 (PKB beta). Science 2001; 292:1728-1731.

10 Fletcher LM, Welsh GI, Oatey PB, Tavare JM. Role for the microtubule cytoskeleton in GLUT4 vesicle trafficking and in the regulation of insulin-stimulated glucose uptake. Biochem $J$ 2000; 352(Part 2):267-276.

11 Huang J, Imamura T, Babendure JL, Lu JC, Olefsky JM. Disruption of microtubules ablates the specificity of insulin signaling to GLUT4 translocation in 3T3-L1 adipocytes. $J$ Biol Chem 2005; 280:42300-42306.

12 Hirokawa N, Noda Y, Tanaka Y, Niwa S. Kinesin superfamily motor proteins and intracellular transport. Nat Rev Mol Cell Biol 2009; 10:682-696.

13 Emoto M, Langille SE, Czech MP. A role for kinesin in insulin-stimulated GLUT4 glucose transporter translocation in 3T3-L1 adipocytes. J Biol Chem 2001; 276:10677-10682.

14 Fletcher LM, Welsh GI, Oatery PB, Tavare JM. Role for the microtubule cytoskeleton in GLUT4 vesicle trafficking and in the regulation of insulin-stimulated glucose uptake. Biochem $J$ 2000; 352:267-276.

15 Olson AL, Trumbly AR, Gibson GV. Insulin-mediated GLUT4 translocation is dependent on the microtubule network. $J$ Biol Chem 2001; 276:10706-10714.

16 Molero JC, Whitehead JP, Meerloo T, James DE. Nocodazole inhibits insulin-stimulated glucose transport in 3T3-L1 adipocytes via a microtubule-independent mechanism. J Biol Chem 2001; 276:43829-43835.

17 Semiz S, Park JG, Nicoloro SM, et al. Conventional kinesin KIF5B mediates insulin-stimulated GLUT4 movements on microtubules. EMBO J 2003; 22:2387-2399.

18 Imamura T, Huang J, Usui I, Satoh H, Bever J, Olefsky JM. Insulin-induced GLUT4 translocation involves protein kinase C-lambda-mediated functional coupling between Rab4 and the motor protein kinesin. Mol Cell Biol 2003; 23:4892-4900.

19 Chiang YJ, Hsiao SJ, Yver D, et al. tankyrase 1 and tankyrase 2 are essential but redundant for mouse embryonic development. PLoS One 2008; 3:e2639.

20 Chi NW, Lodish HF. tankyrase is a golgi-associated mitogenactivated protein kinase substrate that interacts with IRAP in GLUT4 vesicles. J Biol Chem 2000; 275:38437-38444.

21 Keller SR, Scott HM, Mastick CC, et al. Cloning and characterizatin of a novel insulin-regulated membrane aminopeptidase from Glut4 vesicles. J Biol Chem 1995; 270:2361223618.

22 Keller SR, Davis AC, Clairmont KB. Mice deficient in the insulin-regulated membrane aminopeptitase show substantial decreases in glucose transporter GLUT4 levels but maintain normal glucose homeostasis. J Biol Chem 2002; 277:17677- 
17686.

23 Jordens I, Molle D, Xiong W, Keller SR, McGraw TE. Insulinregulated aminopeptidase is a key regulator of GLUT4 trafficking by controlling the sorting of GLUT4 from endosomes to specialized insulin-regulated vesicles. Mol Biol Cell 2010; 21:2034-2044.

24 Yeh TY, Sbodio JI, Tsun ZY, Luo B, Chi NW. Insulin-stimulated exocytosis of GLUT4 is enhanced by IRAP and its partner tankyrase. Biochem J 2007; 402:279-290.

25 Yeh TY, Beiswenger KK, Li P, et al. Hypermetabolism, hyperphagia, and reduced adiposity in tankyrase-deficient mice. Diabetes 2009; 58:2476-2485.

26 Chiang YJ, Nguyen ML, Gurunathan S, et al. Generation and characterization of telomere length maintenance in tankyrase 2-deficient mice. Mol Cell Biol 2006; 26:2037-2043.

27 Pezzolesi MG, Nam M, Nagase T, et al. Examination of candidate chromosomal regions for type 2 diabetes reveals a susceptibility locus on human chromosome 8p23.1. Diabetes 2004; 53:486-491.

28 Li Z, Francois F, Tong Z, et al. The mouse Fused locus encodes Axin, an inhibitor of the Wnt signaling pathway that regulates embryonic axis formation. Cell 1997; 90:181-192.

29 Rui, Y, Xu Z, Xiong B, et al. A beta-catenin-independent dorsalization pathway activated by Axin/JNK signaling and antagonized by Aida. Dev Cell 2007; 13:268-282.

30 Rui Y, Xu Z, Lin S, et al. Axin stimulates p53 functions by activation of HIPK2 kinase through multimeric complex formation. EMBO J 2004; 23:4583-4594.

31 Li Q, Wang X, Wu X, et al. Daxx cooperates with the Axin/ HIPK2/p53 complex to induce cell death. Cancer Res 2007; 67:66-74.

32 Li Q, Lin S, Wang X, et al. Axin determines cell fate by controlling the p53 activation threshold after DNA damage. Nat Cell Biol 2009; 11:1128-1134.

33 Yamazaki H, Nusse R. Identification of DCAP, a drosophila homolog of a glucose transport regulatory complex. Mech Dev 2002; 119:115-119.

34 Huang SM, Mishina YM, Liu S, et al. Tankyrase inhibition stabilizes axin and antagonizes Wnt signalling. Nature 2009; 461:614-620.

35 Bae SS, Cho H, Mu J, Birnbaum MJ. Isoform-specific regula- tion of insulin-dependent glucose uptake by Akt/protein kinase B. J Biol Chem 2003; 278:49530-49536.

36 Gonzalez E, McGraw TE. Insulin signaling diverges into Aktdependent and -independent signals to regulate the recruitment/docking and the fusion of GLUT4 vesicles to the PM. Mol Biol Cell 2006; 17:4484-4493.

37 Sano H, Kane S, Sano E, et al. Insulin-stimulated phosphorylation of a Rab GTPase-activating protein regulates GLUT4 translocation. J Biol Chem 2003; 278:14599-14602.

38 Kane S, Sano H, Liu SC, et al. Akt phosphorylates a novel adipocyte protein with a Rab GTPase-activating protein (GAP) domain. J Biol Chem 2002; 277:22115-22118.

39 Zeigerer A, McBrayer MK, McGraw TE. Insulin stimulation of GLUT4 exocytosis, but not its inhibition of endocytosis, is dependent on RabGAP AS160. Mol Biol Cell 2004; 15:44064415.

40 Miinea CP, Sano H, Kane S, et al. AS160, the Akt substrate regulating GLUT4 translocation, has a functional Rab GTPaseactivating protein domain. Biochem $J$ 2005; 391:87-93.

41 Kramer HF, Witczak CA, Taylor EB, Fujii N, Hirshman MF, Goodyear LJ.. AS160 regulates insulin- and contraction-stimulated glucose uptake in mouse skeletal muscle. J Biol Chem 2006; 281:31478-31485.

42 Thong FS, Bilan PJ, Klip A. The Rab GTPase-activating protein AS160 integrates Akt, protein kinase C, AMP-activated protein kinase signals regulating GLUT4 traffic. Diabetes 2007; 56:414-423

43 Nishiumi S, Ashida H. Rapid preparation of a plasma membrane fraction from adipocytes and muscle cells: application to detection of translocated glucose transporter 4 on the plasma membrane. Biosci Biotechnol Biochem 2007; 71:2343-2346.

(Supplementary information is linked to the online version of the paper on the Cell Research website)

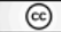

This work is licensed under the Creative Commons Attribution-NonCommercial-No Derivative Works 3.0 Unported License. To view a copy of this license, visit http:// creativecommons.org/licenses/by-nc-nd/3.0 